\title{
Wireless Network Virtualization
}

\author{
(Invited Position Paper) \\ Xin Wang, Prashant Krishnamurthy, and David Tipper \\ Graduate Telecommunications and Networking Program \\ University of Pittsburgh, Pittsburgh, PA 15260 \\ \{xiw54, prashk, dtipper\}@pitt.edu
}

\begin{abstract}
Virtualization of wired networks and end computing systems has become one of the leading trends in networked ICT systems. In contrast relatively little virtualization has occurred in infrastructure based wireless networks, but the idea of virtualizing wireless access is gaining attention as it has the potential to improve spectrum utilization and perhaps create new services. In this paper we survey the state of the current research in virtualizing wireless networks. We define and describe possible architectures, the issues, hurdles and trends towards implementation of wireless network virtualization.
\end{abstract}

\section{INTRODUCTION}

The term "Virtualization" refers to the creation of a set of logical architectures using a given set of physical entities, but in a manner that is transparent to the user. For example, a physical server comprised of processors, memory, network interface cards, and storage may be used to create a set of "virtual" servers that all employ the physical hardware, but the users see these virtual servers as separate entities by themselves. The challenge in this case is to allocate the physical entities to the virtual entities in a way that maximizes utilization of the physical entities while providing the required performance to the user. Ideally, such an allocation should be dynamic (not a one-time allocation) depending on the needs of users. Further, the allocation process should not be cumbersome or resource intensive by itself. The reasons for virtualization are increased hardware efficiencies, easier migration to newer products or technologies while supporting legacy products, and overall reduced cost of equipment and management. Virtualization has occurred in wired networks for decades with Virtual Private Networks (VPNs) over service provider networks common in WANs and MANs and with VLANs in enterprise networks. In general the virtualization is achieved by logically partitioning a physical network into virtual networks that share the physical routers/switches/crossconnects, physical links, and bandwidth on each link. The utilization of the physical resources needs to be carefully managed to maintain the quality of service (QoS) and security needs of the users of each virtual network. In the case of WANs and MANs the process of set up/tear down and management of virtual networks is tightly controlled by service providers.

When virtualization is applied to wireless networks, things quickly become fairly complicated. Wireless network virtualization includes both infrastructure sharing and spectrum sharing. Furthermore, there are many different topologies for wireless networks (infrastructure and ad hoc, and within ad hoc, single and multi-hop), different spectrum bands ranging from several hundred $\mathrm{MHz}$ to several $\mathrm{GHz}$, unlicensed versus licensed spectrum bands, different geographic coverage (wide, metro, local, and personal area) and finally, different mobility requirements. When wireless networks are deployed, the interference that is caused within an administrative unit (e.g., one service provider's network) and across administrative units becomes important. Physical entities in wireless networks can be as diverse as the complex mobility management entity in 4G cellular networks to inexpensive access points in $\mathrm{Wi}-\mathrm{Fi}$ networks. Furthermore, the air-interface and bandwidth slices employed by different technologies can be very different. The protocols on the air (access) and in the backbone (core) networks can be very different across technologies. Also, it is worth noting that governments heavily regulate the basic resource of spectrum. Thus far, there is no unified vision of what wireless network virtualization means and how it may actually be implemented. There have been recent attempts to carve out areas where virtualizing wireless networks seems to be possible, albeit in a restricted manner. This work has been motivated by two different research activities over the last decade, namely (a) the work on dynamic spectrum access and (b) the work on LTE and LTE-Advanced that is unifying the air-interface aspects of wide area cellular services. Also, some work has appeared looking at virtualizing wireless local area and mesh networks.

In this paper, we present our view of wireless network virtualization. We provide some background of recent work in this area in Section II. In Section III, we describe our three wireless network virtualization paradigms. Section IV considers the challenges and hurdles in implementation of virtualization in wireless networks. Lastly, Section V concludes the paper.

\section{BACKGROUND}

Recently there has been some work that has started a discussion of wireless network virtualization. We can classify such discussions based on whether the origins are rooted in (a) dynamic spectrum access for in cellular networks where mobile virtual network operators (MVNOs) are prevalent or (b) based on the technologies considered - cellular vs. Wi-Fi in infrastructure or ad hoc modes. In this section, we briefly discuss some of this work.

\section{A. DSA and MVNO Approaches}

1) Work Originating in DSA: Cognitive radios have been considered as a promising option to access (licensed) spectrum that is not spatio-temporally utilized. In some ways, the use of 
CRs with dynamic spectrum access may be viewed as a type of spectrum virtualization. In a $\mathrm{CR}$ environment, unlicensed or secondary users may sense and access the licensed bands on a negotiated or an opportunistic basis. This is a user-centric paradigm, where the $\mathrm{CR}$ does everything in a localized manner. However, it is very difficult to address technical problems such as hidden nodes with DSA. Even if a CR user $A$ detects a certain portion of the spectrum available and then starts sending signals, $A$ 's transmission may still interfere with radio transmissions of other secondary users or of the primary user since radio propagation is unpredictable as are the locations of other users.

In order to coordinate a large number of CRs, some research papers have integrated brokers into the telecommunication business model [1], [2]. Such dynamic frequency brokers (DFBs) are responsible for allocating frequency bands to radio nodes within their geographic area. Radio nodes submit reports on channel conditions, QoS requirements etc. to DFBs every time interval. DFBs work in a hierarchical manner, with national level DFBs on top of the regional level DFBs. The frequency band allocation is enforced from top to bottom [3]. Bidding procedures (between users and service providers (SPs), or between SPs and spectrum brokers) have been modeled based on game theory in the literature [3], [4], [5]. In this model, no barriers or obstacles are placed for the utilization of spectrum across the entire bandwidth. The spectrum pool is drained based on users demand. In other words, the wireless network evolves into a virtual environment with the presence of DFBs. Spectrum users run their operations without knowing the underlying architecture. However, virtualization of wireless networks, as described later in this paper, is an even broader concept than the DFB system. Virtualization needs not be applied in a user-centric network nor be associated with CRs.

2) MVNOs: A Mobile Virtual Network Operator (MVNO) is a special network operator who leases radio access from a host mobile network operator (MNO). An MVNO can be viewed as a special implementation of wireless virtualization. The strict definition of an MVNO differs from country to country [6]. Typically a MVNO leases spectrum from one or more MNOs, and connects its own subscribers to its own switching center. Alternatively, the network operated by an MVNO may also be connected to the MNOs networks that have agreements with the MVNO. The key distinction between a MVNO and a MNO is a MVNO does not own any spectrum and radio access networks for its subscribers to access. In some countries, regulators may require MNOs to open the networks to MVNOs to enhance competition. On the other hand, MNOs may also make voluntary agreements to engage with MVNOs to derive some benefits. For instance, MVNOs can reach or test new market segments, utilize spare network capacity, and introduce new services which can supplement existing services provided by an MNO [7].

Although the MVNO concept may bring about much needed service differentiation to a cellular network, it is still not a model of complete virtualization for the overall wireless network. MVNOs lease a fixed amount of spectrum from
MNOs. Currently, the radio resources in the access network are not dynamically shared among multiple MVNOs or across MNOs in a fine granularity. This approach has been suggested with LTE as discussed in the next section.

\section{B. Technology Oriented Approaches}

1) LTE Based: The use of LTE for virtualization has been recently explored in the research literature. The idea here is similar to router/switch virtualization in wired networks. The work in [8], [9], [10] proposed an entity called a "Hypervisor" on top of the physical layer in the base stations in LTE (called e-NodeB's or eNBs). The hypervisor virtualizes the eNB into a number of virtual eNBs (each of which is managed by a virtual operator). The hypervisor also allocates the air interface resources (called physical resource blocks or PRBs) among multiple virtual eNBs. Virtual operators share the LTE spectrum based on QoS criteria and give feedback to the hypervisor in each time unit. The hypervisor collects information from individual virtual eNB stacks, such as user channel conditions, traffic loads, priorities, QoS requirements and information related to the contract of each virtual operator [8]. Based on this information, the hypervisor can schedule the air interface resources among multiple virtual networks every time unit. Different configuration methods [8], [9], [10] can be used to complete the scheduling. Multiplexing gains have been reported based on simulations of such virtual networks.

2) WLAN Based: The virtualization of a WLAN access point has been considered in [11]. Rather than pooling spectrum, this work considers resource partitioning of limited spectrum in an optimal and fair way. To this end, the authors manipulate in each virtual WLAN, the contention window in the CSMA/CA based medium access control protocol in IEEE 802.11 WLANs. The complexity of embedding a virtual network over a physical wireless mesh network is studied in [12].

\section{Wireless Network Virtualization Paradigms}

Generally, network virtualization, irrespective of whether it is in wired or wireless environments, can be viewed as splitting the entire system. It is possible to view the network as being composed of Infrastructure Providers or InP's that create and manage only the infrastructure and Service Providers or SPs, which actually provide various services to subscribers. The resources that belong to one or more InPs are virtualized and split into slices. A SP requires a minimum of one slice of the resources from an InPs and provides end-to-end services to end-users, without knowing the underlying physical architecture of the InP. After splitting the resources into slices, each slice creates an illusion that it is an entire system by itself. This "slice" system consists of its own (virtualized) core network and (virtualized) access network corresponding to the wired slice and the wireless slice, respectively.

In recent research work, various analytical and experimental models have been proposed to depict wireless virtualization and evaluate virtual architectures [13], [4], [5], [8], [9], [10], [14]. On the one hand, work that focuses on market profit views a virtual wireless network simply as a spectrum pool 
with hierarchical DFB management as described previously. In such cases, two types of interactions are studied - between users and SPs or between SPs and InPs. Such interactions usually are modeled as stochastic games. The existence of the Nash equilibrium [4] can result in an optimum price for spectrum. On the other hand, research that focuses on implementation of wireless virtualization pick a particular platform like LTE or Wi-Fi. Such works consider case studies and run simulations to evaluate the technical benefits of virtualization. Compared to the work that looks at spectrum pools, the related work in the technical implementation is rather limited. Also, a few works aim at virtualizing one BS to fit the requirements of multiple MVNOs. Some optimization techniques like weighted slice allocation are integrated within the physical BS to create opportunities for MVNOs. MVNOs can then customize their own virtual BSs [14]. However, the MVNOs will need to be able to virtualize the backhaul network and it's components (signaling, mobility management, security functions, etc. ) as well. In a nutshell, even the understanding of what wireless virtualization means is not clear in the literature.

Inspired by the different degrees of virtualization, this paper proposes three paradigms for wireless network virtualization employing the idea of InPs and SPs, namely: (1) universal, (2) cross-infrastructure, and (3) and limited intra-infrastructure.

3) Universal Virtualization: : A grand vision view of wireless network virtualization is to make no assumptions whatsoever about InPs or SPs. This view of wireless network virtualization looks at the whole path of radio access as an "unbundled cloud" where virtualization is pervasive. The cloud is comprised of heterogeneous base stations (macro-cells, picocells, and femto-cells, relays, and other kinds of points of access and wired backbones) that are transparent to the user [5]. It is the responsibility of a provider of a specific service to choose a package of network components, links, and spectrum and the provider configures them in the way it desires. Ideally, this could happen dynamically in an on-demand type fashion. For example, to support a specific application such as one that involves extremely low power transmissions at low rates with not very stringent delay constraints, the network components to be used may be femto-cells using a small slice of spectrum or even sensor relays that use multiple hops to a destination. This "cloud" like virtualization has complicated management, control and economic issues that have not been considered in the literature. For example, how much and what type of management capabilities are given to a SP on InP system or how can mandated/regulated services like E-911 localization be ensured.

4) Cross-infrastructure Virtualization: : In this paradigm we assume that wireless virtualization is possible across InPs (inter-InP) and within InPs. This enables all of the InPs in a geographical area to allow their network resources to be shared across SPs. A simplified example is depicted in Figure 1. In this example, base stations (BS) 1 and 2 belong to InP 1 while BSs 3 and 4 belong to InP 2. Two SPs are in the system SP $A$ and SP $B$. A centralized management has to be implemented to ensure the co-operation and isolation between InPs (for this purpose, an entity named Resource Management is added on top of the InPs). Notice that an InP might have bandwidth slices that support multiple radio access technologies (RATs) such as, GSM, UMTS and LTE. InterInP virtualization allows spectrum sharing between different SPs, different RATs and different InPs. InPs that cover the same region (in Figure 1, BSs $1 \& 3$ or BSs $2 \& 4$ ) provide their physical resources to SPs. SPs are allocated specific resources based on their requirements, every specific time unit. There are no clear boundaries between multiple network infrastructures belonging to different InPs. It is as if all the resources are in the same pool for SPs to employ (e.g., in Figure 1, frequencies $f_{1 A}, f_{1 B}, f_{3 A}, f_{3 B}$ are in the same pool). SPs might choose the resource with the best quality or with the lowest price. However, inter-InP wireless virtualization has strict coverage/interference requirements. The coverage of InPs should either completely overlap or there has to be a way of determining what BSs from which InP covers what part of a geographical area. Otherwise there may be "service holes" when users enter an area which is not covered by a set of InPs used by an SP. Due to the limited wireless coverage of each cell, this virtualization design might be more suitable for certain areas (e.g., urban) that have highly overlapping multiple cells. Not only are the radio resources shared among different SPs, but also the nodes and links, which connect the access network to the core network. Such nodes and links should be shared in a virtualized fashion.

To design an appropriate cross-infrastructure virtualization strategy, several factors have to be taken into account, such as the entire network architecture, the QoS promised by each SP, and the fluctuation of traffic. DFB management can help with the interference mitigation and allocation of spectrum resources. For cross-infrastructure wireless virtualization, a completely centralized management may be preferable. A well-designed centralized strategy will have a higher probability of bringing significant improvement to the network utilization, reliability, and quality of service. But a bad strategy might encroach upon some SPs deserved resource, and such an SPs may not be able to ensure a level of QoS for its users, especially the ones at the edges of coverage.

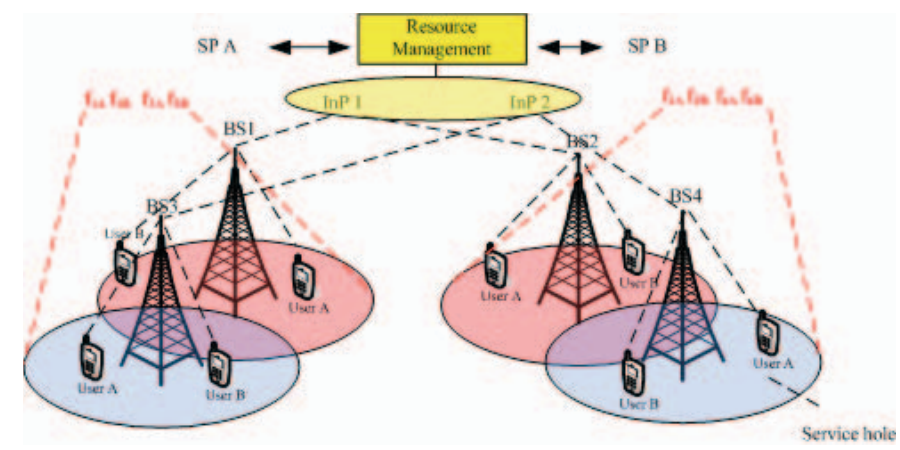

Fig. 1. Cross-Infrastructure Wireless Network Virtualization

5) Limited intra-infrastructure virtualization: : Limited wireless virtualization in our view considers only virtualization 
within a single InP, which may still have spectrum that is used by different RATs. Spectrum sharing occurs between SPs and across RATs. For a given cell, we can think of a single InP that can manage its resources and make decisions to allocate them to various SPs. The multiplexing gains are likely to be lower than those possible with a cross-infrastructure strategy as there may be InPs with demand from SPs that is greater than they can meet while other InPs have resources that are not being completely utilized. Limited virtualization can be described by the example shown in Figure 2. In cell 1 of a cellular system, two SPs $A$ and $B$ lease a certain amount of resource from BS 1 in each time interval. BS 1 is virtualized and in charge of the spectrum $f_{1 A}$ and $f_{1 B}$ allocated to SPs. Compared to a static cellular system, $f_{1 A}$ is no longer exclusive for SP $A$ s users, as also $f_{1 B}$. Every SP can be viewed as a virtual operator (VO) with time-varying resources based on factors such as its own requirement, the amount of money it is willing to pay for resources, fairness, and other InP policies. This is similar to the single-level DFB structure where we can consider the InP as a DFB that assigns spectrum to nodes in its region and SPs as those nodes.

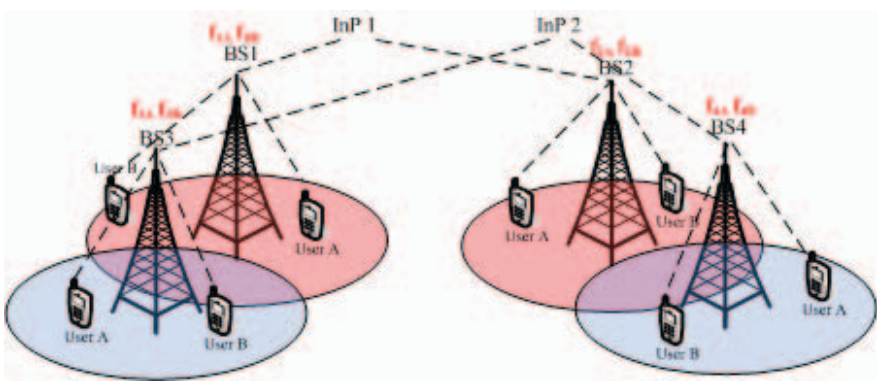

Fig. 2. Limited Intra-Infrastructure Wireless Network Virtualization

\section{ISSUES FOR DISCUSSION}

\section{A. Technical Challenges}

There are several challenges that arise irrespective of the wireless network virtualization paradigm adopted (although the specifics are likely to vary in the degree of complexity). As an extension of wired network virtualization, technical challenges in terms of instantiation, operation and management of wireless network virtualization need to be better explored [15]. Most existing work focuses on spectrum allocation models, for example, some theories like auction game winner determination problems are used to model spectrum allocation [4], [5]. A few experiments have been done on hardware testbeds [16], [17]. However, important issues like interfacing, signaling, mobility management and isolation have not had much attention in the research literature. In this subsection, these issues will be discussed as open challenges mapped from the wired perspective presented in [15].

1) Interfacing: Wired virtualization requires virtual networks to express their needs in terms of virtual nodes and virtual links in a standard specification language [15]. In wireless virtualization, SPs require radio resources (bandwidth, power, interference) from one or more InPs. Since various RATs might be used by SPs on the same InP, a well-defined common interface is a must for InPs to understand the radio resources required by SPs. Furthermore, with multiple InPs, the need for a standard language to express explicit sharing information to each other arises. The communication between SPs and between end-users and SPs also need to be standardized.

2) Signaling and Bootstrapping: A SP must have network connectivity to one or more InPs in order to issue its requests before a virtual network is created. Signaling must be handled properly (in terms of delays and reliability) to enable the InPs or the hypervisor (an entity in InP in charge of coordinating the resources) to enable configuring the network it supports. A bootstrapping capability to allow SPs to customize the virtual resources allocated to them is needed. Wireless virtualization may need out-of band wired or wireless communications for these functions. If all of the spectrum is virtualized, at least a piece of it will may to be dedicated for signaling and bootstrapping unless wired links exist to handle these functions.

3) Resource Allocation: A well known wired virtual network problem is how to embed a virtual network in a physical network (i.e., what nodes, links, and resources should be picked and what should be optimized)[15], and it is also important in wireless virtualization [12]. Embedding of virtual networks, with constraints on resources or requirements, can be reduced to an NP-hard optimization problem [18]. In market-oriented analyses, the problem usually aims to maximizing the revenue of each InP with finite spectrum and SPs QoS requirements as constraints [5]. In cross-infrastructure virtualization, constraints such as finite radio resources, SPs QoS requirements, and different InPs policies need to be included in the problem.

4) Resource Discovery: In order to allocate resources to SPs, InPs or hypervisors should be aware of the available radio resources of the wireless network. Coordination among InPs should be done before each InP assigns its resources to SPs. InPs may need to reserve some resources for themselves in which case the InPs need to determine what radio resources to keep and how much they are willing to share. Resource discovery and allocation create another important issue for network management the transmission time interval (TTI) or time unit between each discovery and allocation of resources. It is obvious that the cost will be astounding if the period is short. But a low update frequency (e.g., monthly SLAs) might drag the network back to the traditional static architecture.

5) Isolation: Compared to wired networks, the performance of wireless networks is much more sensitive to interference. It makes isolation between different users or SPs crucial. In [14], a slice isolation engine (SIE) is used to limit slice traffic irrespective of the clients and services classes agreed before. Another way to deal with isolation is to embed it to the spectrum allocation problem. It can be considered as a constraint in terms of the particular distance between paired spectrum channels [5] for frequency duplexed systems.

6) Mobility Management: Mobile users should be able to smoothly switch to their contracted SP. An even better scenario 
is that users might access any SP either offering the best QoS or the lowest cost in that location. Wireless virtualization should facilitates this mobility management through spectrum/infrastructure sharing and protocols between SPs and InPs to ensure that users can access the most appropriate SP.

7) System Operation: Wireless virtualization may require all InPs to share their physical resources. This scheme potentially allows certain InPs to shut down their equipment when the traffic is lower than some threshold. If the coverage of several InPs overlaps, or the demand is low, it may be possible to save cost by carefully shutting down some of the BSs and sharing the resources of the other BSs. BSs may need extra hardware and software enablers to adapt to the increased spectrum/RAT capabilities. Such system operations have to be reconciled with resource discovery, allocation, isolation, etc.

\section{B. Limitations of Wireless Virtualization}

1) Finite Resources: Unlike cloud computing, the economies of scale that makes virtualization a viable model may not be always applicable to the wireless domain. Coverage in rural areas is often a problem. A smaller number of BSs with limited capabilities in rural areas may not leave enough resources remaining to be shared making virtualization meaningless. Even though the amount of spare resources may be larger than in urban areas, the spread is uneven in geographic terms. Furthermore, spectrum is a regulated resource that cannot easily added to a specific geographic location in contrast to cloud computing where additional computing resources can be quickly added.

2) End Device: Though wireless virtualization may not require major changes in existing BSs, end devices have to be adapted to be able to access a broader range of carrier frequencies. Flexible spectrum sharing needs enablers, for instance, frequency agile broadband radios and direct conversion architectures. An end device needs to be equipped with hardware to enable itself to access the entire frequency band. Software to compute the spectrum sharing algorithms should also be available.

\section{Non-technical Challenges}

Technically, it appears that the potential multiplexing gains and better spectrum utilization are good reasons for wireless network virtualization. However, wireless network virtualization is unlikely to happen in practice and may suffer the same fate as many other promising but unsuccessful technologies without a good economic rationale and a friendly regulatory environment. One of the few papers that illustrate a use-case for a (limited) virtualized wireless network is the work in [19], which uses enterprise cloud access by mobile devices as a motivating example. In terms of regulation, the ownership of spectrum, physical infrastructure, and provision of services will likely have to be unbundled. It is unlikely that legacy services will be willing to easily share their resources unless strong economic and regulatory reasons arise. Further, we have only considered two-way communications implicitly in this paper. For all of the useful spectrum to be virtualized, oneway broadcast communications will also have to be considered and the support for legacy devices carefully examined.

\section{CONCLUSION}

Providing virtualization in wireless networks is a promising technological concept that has the potential to relieve spectrum congestion and open new services. In this paper we discussed three paradigms for virtualizing wireless networks: (1) universal, (2) cross-infrastructure and (3) limited intra-infrastructure. The paradigms vary in the degree of virtualization and infrastructure sharing. Each paradigm incurs technical and nontechnical hurdles that must be overcome before wireless virtualization becomes a widespread technology. These challenges do not mean wireless virtualization is impossible but that it requires careful design and evaluation.

\section{REFERENCES}

[1] T. Maseng and T. Ulversoy, "Dynamic frequency broker and cognitive radio," The IET Seminar on Cognitive Radio and Software Defined Radios: Technologies and Techniques, September 2008.

[2] G. Isiklar and A. Bener, "Brokering and pricing architecture over cognitive radio wireless networks," Proc. IEEE CCNC, January 2008.

[3] M. M. Buddhikot, P. Kolodzy, S. Miller, K. Ryan, and J. Evans, "Dimsumnet: New directions in wireless networking using coordinated dynamic spectrum," WoWMoM, pp. 78-85, 2005.

[4] F. Fu and U. C. Kozat, "Wireless network virtualization as a sequential auction game," Proc. IEEE Infocom, 2010.

[5] T. K. Forde et al., "Exclusive sharing and the virtualization of the cellular network," Symposium on Dynamic Spectrum Access Networks, 2011.

[6] B. W. Kima and S. H. Seol, "Economic analysis of the introduction of the mvno system and its major implications for optimal policy decisions in korea," Telecommunications Policy, vol. 31, no. 5, pp. 290-304, 2007.

[7] ., "Mvno pricing structures in finland," Finnish Ministry of Transport and Communications, Helsinki, 2005., Tech. Rep., 2005.

[8] Y. Zaki, L. Zhao, A. Timm-Giel, and C.Gorg, "A novel lte wireless virtualization framework," Second International ICST Conference on Mobile Networks And Management, 2010.

[9] —_, "Lte wireless virtualization and spectrum management," Third Joint IFIP Wireless and Mobile Networking Conference (WMNC), 2010.

[10] Y. Zaki, L. Zhao, C.Gorg, and A. Timm-Giel, "Lte mobile network virtualization," Springer Mobile Networks and Applications, vol. 16, no. 2, pp. 424-432, August 2011.

[11] A. Banchs, P. Serrano, P. Patras, and M. Natkaniec, "Providing throughput and fairness guarantees in virtualized wlans through control theory," Springer Mobile Networks and Applications, 2012.

[12] D. Yun and Y. Yi, "Virtual network embedding in wireless multihop networks," Proceedings of ACM CFI, June 13-15 2011.

[13] H. Anouar, C. Bonnet, D. Câmara, F. Filali, and R. Knopp, "An overview of open air interface wireless network emulation methodology," $A C M$ Sigmetrics Performance Evaluation Review, vol. 36, no. 2, September 2008.

[14] G. Bhanage, I. Seskar, R. Mahindra, and D. Raychaudhuri, "Virtual basestation: architecture for an open shared wimax framework," Proceedings of the second ACM SIGCOMM workshop on Virtualized infrastructure systems and architectures, September 2010.

[15] N. M. K. Chowdhury and R. Boutaba, "A survey of network virtualization,” Elsevier Computer Networks, vol. 54, pp. 862-876, 2010.

[16] Y. He, J. Fang, J. Zhang, H. S. K. Tan, and Y. Zhang, "Mpap: Virtualization architecture for heterogenous wireless aps." CCR Online, 2010.

[17] S. Singhal, G. Hadjichristofi, I. Seskar, and D. Raychaudhuri, "Evaluation of uml based wireless network virtualization." In Next Generation Internet (NGI) testbeds, 2008.

[18] D. Andersen. (2002) Theoretical approaches to node assignment. [Online]. Available: http://www.cs.cmu.edu/dga/ papers/andersenassign.ps

[19] A. Baliga, X. Chen, B. Coskun, G. de los Reyes, S. Lee, S. Mathur, and J. E. V. der Merwe, "Vpmn - virtual private mobile network towards mobility-as-a-service," Proceedings of ACM MCS, 2011. 